\title{
Diagnosis and Prognosis of Retroperitoneal Liposarcoma: A Single Asian Center Cohort of 57 Cases
}

\author{
Jianchun Xiao $\mathbb{D}^{1},{ }^{1}$ Jianghao Liu $\mathbb{D},{ }^{2}$ Minting Chen $\mathbb{D}^{2},{ }^{2}$ Wei Liu $\mathbb{D}$, ${ }^{1}$ and Xiaodong He ${ }^{1}{ }^{1}$ \\ ${ }^{1}$ Department of General Surgery, Peking Union Medical College Hospital, \\ Chinese Academy of Medical Sciences \& Peking Union Medical College, No. 1 Shuaifuyuan, Beijing 100730, China \\ ${ }^{2}$ Chinese Academy of Medical Sciences \& Peking Union Medical College, Beijing 100005, China \\ Correspondence should be addressed to Wei Liu; liuwei3359@pumch.cn and Xiaodong He; hexd@pumch.cn \\ Jianchun Xiao, Jianghao Liu, and Minting Chen contributed equally to this work.
}

Received 8 July 2020; Revised 23 February 2021; Accepted 28 February 2021; Published 1 April 2021

Academic Editor: Shinji Miwa

Copyright (C) 2021 Jianchun Xiao et al. This is an open access article distributed under the Creative Commons Attribution License, which permits unrestricted use, distribution, and reproduction in any medium, provided the original work is properly cited.

\begin{abstract}
Background. Liposarcoma is a soft tissue malignancy, commonly observed in the extremities. However, retroperitoneal liposarcoma is seldom reported and its diagnosis is frequently neglected. This study aims to present the clinical characteristics, diagnosis, and prognosis of five liposarcoma subtypes and report our experience of patient treatment. Methods. We conducted a single-center noninterventional retrospective study of 57 retroperitoneal liposarcoma patients admitted to Peking Union Medical College Hospital (PUMCH, Beijing, China) between July 2011 and December 2019. We collected and analyzed their demographic, clinical, imaging, histological, therapeutic, and prognostic data over a mean 4.5-year follow-up period. Results. Twenty-five (44\%) patients were asymptomatic prior to diagnosis, with abdominal distension as the chief complaint in 18 (32\%) patients and abdominal pain observed in $16(28 \%)$ patients. Masses were evaluated by computed tomography $(n=48,84 \%)$ or ultrasound $(n=25,44 \%)$. Laparotomy $(n=52,91 \%)$ was the dominant therapeutic modality rather than laparoscopy $(n=5,9 \%)$. All patients were treated with R0 resection except two patients who underwent R2 resection. We conducted regular follow-ups every six months after surgery for a mean duration of 4.5 years. Recurrence was experienced by 14 (25\%) patients and a further 9 (16\%) died during follow-up. Conclusions. Abdominal distension and pain are chief complaints with liposarcoma. As the extremities are the main liposarcomas locations, the diagnosis of retroperitoneal liposarcoma is usually neglected. Since half of the patients are asymptomatic, timely diagnosis and treatment are highly dependent on regular ultrasound and computed tomography imaging. $\mathrm{R} 0$ resection is the key to retroperitoneal liposarcoma treatment. In comparison, patients who underwent R2 resection, which is considered a palliative treatment, had bad prognoses. Large, symptomatic dedifferentiated, and pleomorphic liposarcomas are more likely to have poor prognoses, while the prognosis for well-differentiated or myxoid liposarcoma is relatively good.
\end{abstract}

\section{Introduction}

Accounting for only approximately $10 \%$ of all soft tissue sarcomas and $15 \%$ of all sarcomas, liposarcoma (LPS) is a frequently observed tumor derived from adipocytic differentiated primitive mesenchymal cells. Its incidence peaks in the age range 50-60 years. $[1,2]$. Although it occurs predominantly in the deep soft tissues of the extremities, LPS is also reported in the abdomen in areas such as the esophagus, stomach, and descending mesocolon $[3,4]$. The retroperitoneum is a rare LPS location, with only a few publications discussing its diagnosis, clinical characteristics, and prognosis $[2,5,6]$. LPS is generally classified into five subtypes: well-differentiated LPS (WDLPS), dedifferentiated LPS (DLPS), myxoid LPS (MLPS), pleomorphic LPS (PLPS), and mixed LPS. WDLPS is the most common retroperitoneal LPS, accounting for $40-45 \%$ of all LPS [2, 7-9]. Gene amplification in the 12q12-21 and 10p11-14 chromosomal regions is often observed in WDLPS and DLPS, and DLPS is also associated with at $6 \mathrm{q} 23$ and $1 \mathrm{p} 32[8,10]$. In addition, there is an approximately $10 \%$ probability of WDLPS converting to DLPS, a more invasive LPS subtype [8]. Approximately $95 \%$ of MLPS patients have a $\mathrm{t}(12 ; 16)$ (q13; p11) reciprocal chromosomal translocation resulting in an 
in-frame fusion of the RNA-binding protein fused in sarcoma (FUS) gene to the DNA damage inducible transcript 3 (DDIT3) gene while the remaining $5 \%$ of patients exhibit a $\mathrm{t}(12 ; 22)$ (q13;q12) translocation [8, 11]. To date, no consistent chromosomal or molecular aberration has been reported for PLPS, the rarest of the subtypes [8]. Diagnosis of LPS is currently highly dependent on pathological findings, with computed tomography (CT) or magnetic resonance imaging (MRI) responsible for the majority of presurgical diagnoses $[11,12]$. Since many liposarcomas can be asymptotic before diagnosis, and symptoms, if there are any, are mainly nonspecific such as abdominal pain or distension, the preoperative diagnosis of LPS is difficult [2, 13]. Although the gold standard for diagnosis remains biopsy, imaging is currently a widely accepted diagnostic tool $[2,14]$. The presence of macroscopic fat on imaging suggests the presence of LPS.

Upon diagnosis, surgery is the primary recommended treatment for retroperitoneal LPS; however, the extent of resection remains controversial [15]. Although it is conventional to only resect directly involved adjacent organs, a more aggressive approach, which proposes partial resection of adjacent uninvolved organs, has also been suggested $[15,16]$. Several phase II or III clinical trials have found that chemotherapy, such as trabectedin and eribulin, may improve LPS prognosis $[17,18]$. Immunotherapy for LPS is now also under development.

The prognosis of LPS is highly dependent on the surgical approach and the histological subtype. WDLPS, together with low-grade MLPS, has a 5-year survival rate above $90 \%$. In contrast, the 5-year survival rates of PLPS, DLPS, and high-grade MLPS are all below 75\%, with PLPS showing the lowest of only $50 \%[2,19]$.

\section{Methods}

The medical records of all retroperitoneal LPS patients presenting at Peking Union Medical College Hospital (PUMCH), Beijing, China, between July 2011 and December 2019 were retrospectively reviewed. Informed consent was obtained from each patient. The study was approved by the Institutional Review Board of PUMCH.

Detailed demographic and clinical data, such as histological subtype of tumor, symptoms, and physical signs, from all 57 patients were reviewed. All available imaging examinations, including ultrasound, computed tomography (CT), and MRI, were also collected. In addition, the surgical approach, together with surgical details such as surgery duration and pathological findings, was recorded. To assess the prognosis, the patients were interviewed regularly to obtain details of any relapse, postsurgical chemotherapy, or other adjuvant therapies. Regular follow-ups every six months after surgery, including CT imaging and tumor marker measurements, were conducted for all patients, with the most recent follow-up in February 2020. All data were recorded and analyzed using Python 3.7. Descriptive data were expressed in numbers (\%) for categorical variables and means (SD) for continuous variables, as appropriate. Categorical variables were assessed using Pearson $\chi^{2}$-test or
Fisher's exact test where appropriate. A $t$-test was used to analyze continuous variables. The overall survival and disease-free survival were correlated to symptoms, LPS pathological type, tumor diameter, and organ invasion using the Kaplan-Meier method. All tests were two-sided with $P<0.05$ considered statistically significant.

\section{Results}

A total of 57 patients (26 males and 31 females) suffering from retroperitoneal LPS were recruited from PUMCH. The mean age at treatment was 57.0 (12.2) years. These patients were followed up regularly with the mean duration of followup being 4.5 (2.6) years.

Collation of the history of the current illness demonstrated that $25(44 \%)$ patients had been completely asymptomatic, LPS having been detected during routine medical examinations. Abdominal discomfort was the most common symptom, with 18 (32\%) patients complaining of abdominal distension and 16 (28\%) complaining of abdominal pain, including $2(4 \%)$ who had complained of both. Besides abdominal discomfort, lower extremity symptoms, including swelling and pain, were also reported in $6(11 \%)$ patients. Unsurprisingly, larger tumor size was directly related to complaints of discomfort. For those whose tumor was less than $15 \mathrm{~cm}$, about half (46\%) experienced no abdominal discomfort, whereas only $33 \%$ of patients with a tumor larger than $25 \mathrm{~cm}$ had no abdominal discomfort. Mixed LPS tumors were the largest with an average size of $23.1 \mathrm{~cm}$ while MLPS tumors showed the smallest average size of only $13.1 \mathrm{~cm}$. However, considering the size of our cohort, no solid conclusion can be reached. Besides, the chief complaints differ among the five LPS subtypes. Half of the WDLPS patients and $75 \%$ of the PLPS patients were asymptomatic before diagnosis while 57\% of MLPS patients experienced abdominal distension as their chief complaint, and $48 \%$ of DLPS patients complained of abdominal pain (Table 1).

Detailed physical examination (PE) was conducted on each patient. Only 18 (32\%) among the total of 57 had no apparent positive signs of the disease, the retroperitoneal mass tangible in all the other $39(68 \%)$ patients. Among the 39 patients with tangible mass, 29 (74\%) had little mobility, and $29(74 \%)$ had a clear margin. Tenderness pain was reported by only $14(36 \%)$ patients. As for subtypes, half of WDLPS patients had tangible retroperitoneal masses while the other half did not. Among 23 DLPS patients, retroperitoneal masses of $16(70 \%)$ patients were tangible, and 7 (30\%) were not tangible. All PLPS and mixed LPS patients had tangible retroperitoneal masses (Table 1).

For all 57 patients, retroperitoneal LPS was diagnosed via presurgical imaging and postsurgical pathological analysis. The form of presurgical imaging used for diagnosis included ultrasound (Figure 1), CT (Figure 2), and MRI. All cases of LPS were located within the retroperitoneum and in 7 (12\%) patients the tumor mass had also invaded the pelvis. In 53 (93\%) patients, the tumors were situated in only half of the retroperitoneum, 29 of which were on the left side and 24 on the right side. No family history of LPS was reported in any 
TABle 1: Demographic and clinical characteristics of retroperitoneal LPS patients.

\begin{tabular}{|c|c|}
\hline \multicolumn{2}{|l|}{ Demographic characteristics $n=57$} \\
\hline Sex male/female & $26 / 31$ \\
\hline Age at diagnosis, mean (SD), years & $57.0(12.2)$ \\
\hline Duration of follow-up, mean (SD), years & $5.3(2.6)$ \\
\hline \multicolumn{2}{|l|}{ Clinical characteristics $n=57$} \\
\hline \multicolumn{2}{|l|}{ Location } \\
\hline Retroperitoneum & $57(100 \%)$ \\
\hline Pelvis invasion & $7(12 \%)$ \\
\hline Left retroperitoneum & $29(51 \%)$ \\
\hline Right retroperitoneum & $24(42 \%)$ \\
\hline Both retroperitoneums & $4(7 \%)$ \\
\hline \multicolumn{2}{|l|}{ Symptoms } \\
\hline WDLPS & 18 \\
\hline Asymptomatic & $10(56 \%)$ \\
\hline Abdominal distension & $6(33 \%)$ \\
\hline Abdominal pain & $2(11 \%)$ \\
\hline DLPS & 23 \\
\hline Abdominal pain & $11(48 \%)$ \\
\hline Asymptomatic & $9(39 \%)$ \\
\hline Abdominal distension & $5(21 \%)$ \\
\hline Lower extremity discomfort & $5(21 \%)$ \\
\hline Fever & $2(9 \%)$ \\
\hline Vomiting & $1(4 \%)$ \\
\hline Frequent micturition & $1(4 \%)$ \\
\hline PLPS & 4 \\
\hline Asymptomatic & $3(75 \%)$ \\
\hline Abdominal pain & $1(25 \%)$ \\
\hline MLPS & 7 \\
\hline Abdominal distension & $4(57 \%)$ \\
\hline Asymptomatic & $3(43 \%)$ \\
\hline Abdominal pain & $2(29 \%)$ \\
\hline Mixed LPS & 6 \\
\hline Abdominal distension & $3(50 \%)$ \\
\hline Asymptomatic & $1(17 \%)$ \\
\hline Lower extremity discomfort & $1(17 \%)$ \\
\hline Dysuria & $1(17 \%)$ \\
\hline Overall & 57 \\
\hline Asymptomatic & $25(44 \%)$ \\
\hline Abdominal distension & $18(32 \%)$ \\
\hline Abdominal pain & $16(28 \%)$ \\
\hline Lower extremity discomfort & $6(11 \%)$ \\
\hline Urinary system symptom & $2(4 \%)$ \\
\hline \multicolumn{2}{|l|}{ Physical signs } \\
\hline WDLPS & 18 \\
\hline Absent & $9(50 \%)$ \\
\hline Tangible mass & $9(50 \%)$ \\
\hline DLPS & 23 \\
\hline Absent & $7(30 \%)$ \\
\hline Tangible mass & $16(70 \%)$ \\
\hline PLPS & 4 \\
\hline Tangible mass & $4(100 \%)$ \\
\hline MLPS & 7 \\
\hline Absent & $2(29 \%)$ \\
\hline Tangible mass & $5(71 \%)$ \\
\hline Mixed LPS & 5 \\
\hline Tangible mass & $5(100 \%)$ \\
\hline Overall & 57 \\
\hline Absent & $18(32 \%)$ \\
\hline Tangible mass & $39(68 \%)$ \\
\hline
\end{tabular}

TABLE 1: Continued.

\begin{tabular}{lc}
\hline Characteristics of retroperitoneal mass & \\
WDLPS & 9 \\
Clear border & $8(89 \%)$ \\
Good mobility & $3(33 \%)$ \\
Tenderness pain & $2(22 \%)$ \\
DLPS & 16 \\
Clear border & $9(56 \%)$ \\
Good mobility & $3(19 \%)$ \\
Tenderness pain & $8(50 \%)$ \\
PLPS & 4 \\
Clear border & $4(100 \%)$ \\
Good mobility & $1(25 \%)$ \\
Tenderness pain & $1(25 \%)$ \\
MLPS & 5 \\
Clear border & $5(100 \%)$ \\
Good mobility & $2(40 \%)$ \\
Tenderness pain & $2(40 \%)$ \\
Mixed LPS & 5 \\
Clear border & $3(60 \%)$ \\
Good mobility & $1(20 \%)$ \\
Tenderness pain & $1(20 \%)$ \\
Overall & 39 \\
Clear border & $29(74 \%)$ \\
Good mobility & $10(26 \%)$ \\
Tenderness pain & $14(36 \%)$ \\
\hline Imaging methods & $9 / 11(82 \%)$ \\
B ultrasound & $9 / 11(82 \%)$ \\
CT scan & $2 / 11(18 \%)$ \\
MRI & \\
\hline
\end{tabular}

MLS: myxoid liposarcoma; CT: computed tomography; MRI: magnetic resonance imaging; CM: centimeter; SD: standard deviation.

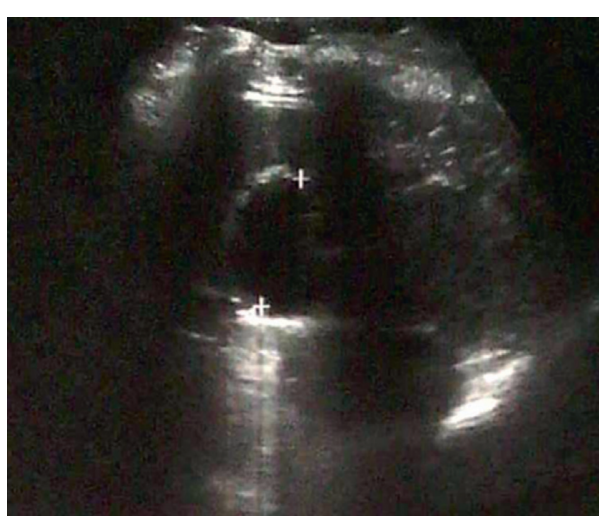

FIgURE 1: Ultrasound of retroperitoneal MLS patient. Retroperitoneal MLS, indicated by while "+" symbols.

of the 57 patients (Table 1). Patients were divided into three subgroups according to maximum tumor diameter indicated by imaging examination (larger than $25 \mathrm{~cm}$, medium, or smaller than $15 \mathrm{~cm}$ ) (Table 2). Certain characteristics observed on ultrasound or CT, such as echoic and density, were not found to be predictive presurgical factors for pathological subtypes: both mixed and low CT densities were reported in each subtype, and no significant differences were observed in the ratios (Table 3). 


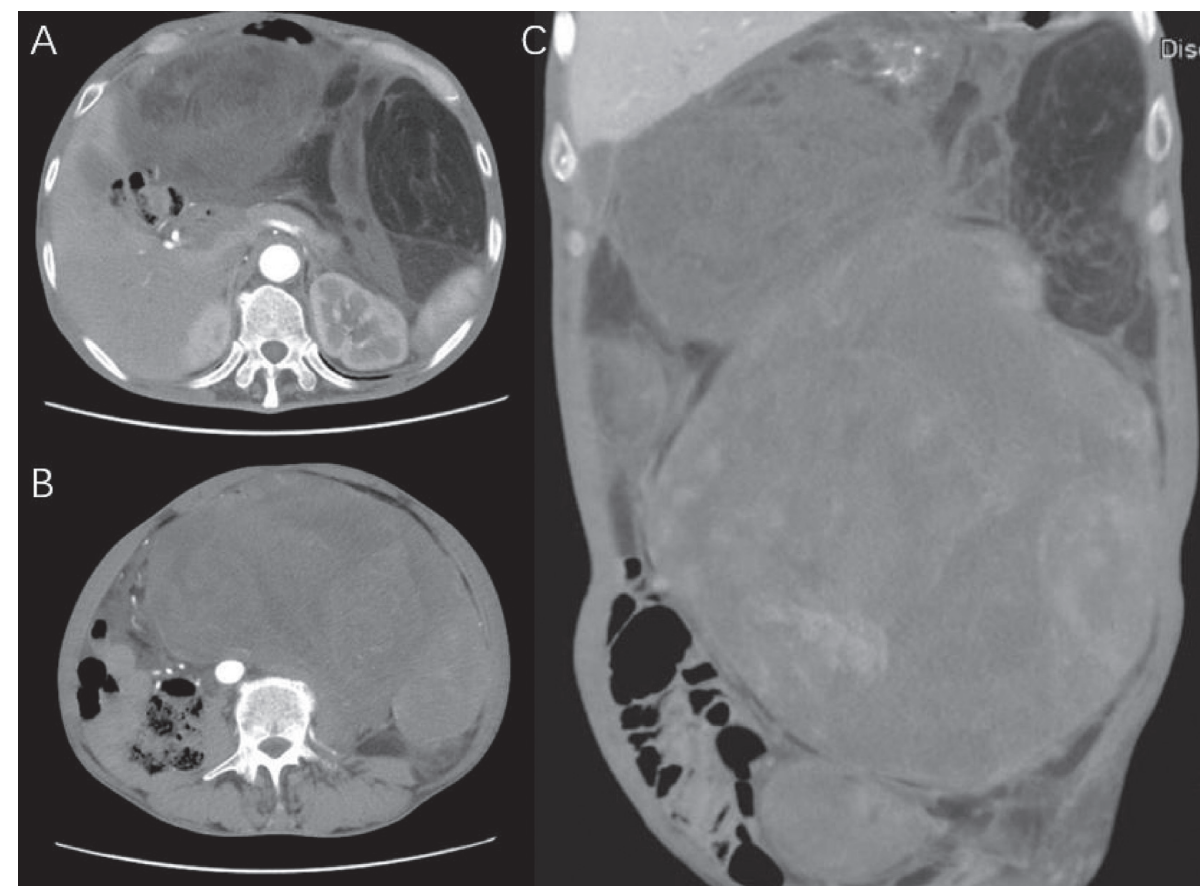

FIgURE 2: CT scan of retroperitoneal MLS patients. (a) Huge retroperitoneal MLS visible at kidney level. (b) Huge retroperitoneal MLS visible at colon level. (c) Huge retroperitoneal MLS, coronal view.

TABLe 2: Size of tumor determined by different methods.

\begin{tabular}{|c|c|c|c|c|c|}
\hline No.* & $\mathrm{PE}$ & $\mathrm{CT}$ & Ultrasound & Resection & Subgroup \\
\hline 1 & $7 * 4$ & $12 * 11$ & NA & $18.5 * 17 * 10.3$ & Small \\
\hline 2 & 10 & NA & $11.6 * 9.6 * 10.4$ & $15 * 8 * 6$ & Small \\
\hline 3 & $10 * 5$ & $10.8 * 5.4$ & $7.8 * 5.9$ & $11 * 7.5 * 6$ & Small \\
\hline 4 & $20 * 9$ & $17.9 * 17.7 * 12.1$ & $15.8 * 11.7$ & $22.8 * 19.5 * 10$ & Middle \\
\hline 5 & Intangible & NA & $10 * 8$ & $20 * 20 * 5.5$ & Small \\
\hline 6 & Intangible & $9.6 * 7.6$ & NA & $12.5 * 8.5 * 6$ & Small \\
\hline 7 & $10 * 10$ & $17.2 * 13$ & No data & $18 * 15 * 9$ & Middle \\
\hline 8 & Intangible & $7.4 * 6$ & NA & $11.4 * 9 * 5.8$ & Small \\
\hline 9 & 10 & $25.2 * 13.7 * 10$ & NA & $30 * 28 * 17$ & Large \\
\hline 10 & $10 * 10$ & 12.5 & $14.9 * 12.9 * 10.7$ & $9 * 9 * 4.8$ & Small \\
\hline 11 & 10 & $12.3 * 11.0 * 8.9$ & NA & $16 * 12 * 6$ & Small \\
\hline 12 & Intangible & $5.7 * 4.9 * 3.8$ & NA & $7.5 * 5 * 4.2$ & Small \\
\hline 13 & $15 * 15$ & $20.6 * 16.1 * 9.7$ & NA & $19 * 16 * 6.5$ & Middle \\
\hline 14 & $25 * 20$ & $20 * 14 * 25$ & NA & $30 * 25 * 10$ & Middle \\
\hline 15 & Intangible & $12.9 * 12.3 * 17.2$ & NA & $19 * 15 * 8$ & Middle \\
\hline 16 & $20 * 15$ & $24.4 * 17.5 * 21.4$ & NA & $22 * 20 * 4.5$ & Middle \\
\hline 17 & 10 & $21.2 * 12.9$ & NA & $25 * 22 * 9.5$ & Middle \\
\hline $18 *$ & Intangible & $34.3 * 31.7 * 23.8$ & NA & $37 * 30 * 16.5$ & Large \\
\hline 19 & 10 & $30 * 30 * 20$ & NA & $21 * 18.5 * 3.5$ & Large \\
\hline 20 & Intangible & $4.2 * 3$ & NA & $5.5 * 3 * 1.5$ & Small \\
\hline 21 & Intangible & $4.5 * 4.5 * 2.7$ & $4.5 * 3.2$ & $4 * 3.5 * 3$ & Small \\
\hline 22 & Intangible & NA & NA & $4.5 * 2 * 2$ & Small \\
\hline 23 & $15 * 15$ & $15.8 * 15.3$ & No data & $20 * 17 * 12$ & Middle \\
\hline 24 & 10 & $10 * 9.7 * 9.6$ & NA & $11 * 9 * 8$ & Small \\
\hline 25 & No data & $26.5 * 13.1 * 36.6$ & No data & $32 * 40 * 20$ & Large \\
\hline 26 & 25 & $24.9 * 23.9 * 28.2$ & NA & $48 * 36 * 12$ & Middle \\
\hline 27 & No data & $23 * 20$ & $25 * 17$ & $33 * 21 * 12$ & Middle \\
\hline 28 & 20 & NA & $25.3 * 18.8 * 14.2$ & $15 * 13 * 3$ & Large \\
\hline 29 & $25 * 15$ & $16 * 9.5$ & NA & $25 * 17 * 9$ & Middle \\
\hline 30 & $18 * 10$ & $18.0 * 12.5 * 11.4$ & $18.6 * 11.6$ & $18 * 12 * 9$ & Middle \\
\hline 31 & No data & $30.5 * 20.9$ & NA & $40 * 35 * 20$ & Large \\
\hline
\end{tabular}


TABLE 2: Continued.

\begin{tabular}{|c|c|c|c|c|c|}
\hline No.* & $\mathrm{PE}$ & $\mathrm{CT}$ & Ultrasound & Resection & Subgroup \\
\hline 32 & 20 & NA & $17.5 * 12.2 * 13.6$ & $30 * 25 * 6$ & Middle \\
\hline 33 & 30 & No data & NA & $38 * 30 * 8.5$ & Large \\
\hline 34 & Intangible & NA & $10.3 * 5.9$ & $13.5 * 11.5 * 8.5$ & Small \\
\hline 35 & $12 * 12$ & $12 * 12$ & NA & $18 * 13 * 7$ & Small \\
\hline 36 & Intangible & $8.6 * 5.5$ & NA & $15 * 11 * 5$ & Small \\
\hline 37 & Intangible & $8.9 * 7.3 * 9.7$ & NA & $11.7 * 9 * 9$ & Small \\
\hline 38 & 10 & $14 * 14 * 16.5$ & NA & $20 * 20 * 12$ & Middle \\
\hline 39 & $10 * 10$ & $14.3 * 9.7$ & NA & $19.5 * 15.8 * 9.5$ & Middle \\
\hline 40 & Intangible & NA & $10 * 7.2$ & $20 * 16 * 5$ & Small \\
\hline 41 & $25 * 15$ & $17.6 * 8.9$ & NA & $15.5 * 8$ & Middle \\
\hline 42 & $12 * 12$ & $12 * 8$ & $11.8 * 9.5 * 9.2$ & $15 * 14 * 14$ & Small \\
\hline 43 & Intangible & $12.2 * 9.9 * 8.5$ & NA & $15 * 12 * 7$ & Small \\
\hline 44 & 25 & $26.4 * 25.4 * 16.5$ & NA & $36 * 32 * 8$ & Large \\
\hline 45 & Intangible & $16 * 13$ & $20.2 * 16.1 * 15.5$ & $23 * 15.5 * 14.5$ & Middle \\
\hline 46 & No data & $7.5 * 5.4 * 6.6$ & NA & $9 * 8.5 * 1.5$ & Small \\
\hline 47 & 15 & $19.2 * 9.3$ & NA & $17 * 14 * 8$ & Middle \\
\hline 48 & $25 * 20$ & NA & NA & $15 * 15 * 10$ & Middle \\
\hline 49 & Intangible & $5.1 * 4.1$ & $5.6 * 4.7$ & $13 * 11 * 5$ & Small \\
\hline 50 & $15 * 5$ & $6.9 * 2.5 * 4.5$ & $13.7 * 18 * 6.2$ & $22.5 * 15 * 7$ & Middle \\
\hline 51 & Intangible & NA & $11.2 * 5.3$ & $15 * 13 * 4$ & Small \\
\hline 52 & $15 * 10$ & No data & NA & $17 * 14 * 12$ & Small \\
\hline 53 & 8 & $13.3 * 12.0 * 27.8$ & $15.3 * 13.2 * 11.2$ & $25 * 14 * 13$ & Large \\
\hline 54 & $20 * 15$ & $24.5 * 12.7 * 23.3$ & $14.7 * 8.48$ & $29 * 16 * 11$ & Middle \\
\hline 55 & Intangible & NA & $9.3 * 5.4$ & $15 * 11 * 3$ & Small \\
\hline 56 & $10 * 10$ & $9.21 * 9.95 * 9.26$ & NA & $16 * 12 * 7$ & Small \\
\hline 57 & $7 * 8$ & $16.5 * 16.2 * 13.7$ & $11.4 * 7.4 * 6.6$ & $16.8 * 9 * 6.5$ & Middle \\
\hline
\end{tabular}

All data are in centimeters. NA: corresponding examination was not performed. No data: corresponding examination was performed, but no specific number was recorded. *No. 18: although large, the tumor mass was intangible, and the inaccessibility of the tumor may be related to hernia.

TABle 3: Characteristics of tumor determined by different methods.

\begin{tabular}{|c|c|c|c|c|}
\hline Patient no. & $\mathrm{PE}$ & Ultrasound-echoic & CT-density & Pathology \\
\hline 1 & Tangible & NA & Low & PLPS \\
\hline 2 & Tangible & Mixed-echoic & NA & PLPS \\
\hline 3 & Tangible & Hypoechoic & Low & PLPS \\
\hline 4 & Tangible & Mixed-echoic & Mixed & PLPS \\
\hline 5 & Intangible & Mixed-echoic & NA & WDLPS \\
\hline 6 & Intangible & NA & Mixed & WDLPS \\
\hline 7 & Tangible & Hyperechoic & Low & WDLPS \\
\hline 8 & Intangible & NA & Low & WDLPS \\
\hline 9 & Tangible & NA & Mixed & WDLPS \\
\hline 10 & Tangible & Hyperechoic & Low & WDLPS \\
\hline 11 & Tangible & NA & Low & WDLPS \\
\hline 12 & Intangible & NA & Mixed & WDLPS \\
\hline 13 & Tangible & NA & Mixed & WDLPS \\
\hline 14 & Tangible & NA & Low & WDLPS \\
\hline 15 & Intangible & NA & Low & WDLPS \\
\hline 16 & Tangible & NA & Low & WDLPS \\
\hline 17 & Tangible & NA & Low & WDLPS \\
\hline 18 & Intangible & NA & Mixed & WDLPS \\
\hline 19 & Tangible & NA & Mixed & WDLPS \\
\hline 20 & Intangible & NA & Low & WDLPS \\
\hline 21 & Intangible & Hypoechoic & Low & WDLPS \\
\hline 22 & Intangible & NA & NA & WDLPS \\
\hline 23 & Tangible & Mixed-echoic & Low & Mixed LPS \\
\hline 24 & Tangible & NA & Mixed & Mixed LPS \\
\hline 25 & Tangible & Hypoechoic & Mixed & Mixed LPS \\
\hline 26 & Tangible & NA & Mixed & Mixed LPS \\
\hline
\end{tabular}


TABle 3: Continued.

\begin{tabular}{|c|c|c|c|c|}
\hline Patient no. & $\mathrm{PE}$ & Ultrasound-echoic & CT-density & Pathology \\
\hline 27 & Tangible & Hypoechoic & Low & Mixed LPS \\
\hline 28 & Tangible & Hyperechoic & NA & DLPS \\
\hline 29 & Tangible & NA & Low & DLPS \\
\hline 30 & Tangible & Hypoechoic & Low & DLPS \\
\hline 31 & Tangible & NA & Mixed & DLPS \\
\hline 32 & Tangible & Hyperechoic & NA & DLPS \\
\hline 33 & Tangible & NA & Mixed & DLPS \\
\hline 34 & Intangible & Hypoechoic & Mixed & DLPS \\
\hline 35 & Tangible & NA & Mixed & DLPS \\
\hline 36 & Intangible & NA & Mixed & DLPS \\
\hline 37 & Intangible & NA & Mixed & DLPS \\
\hline 38 & Tangible & NA & Low & DLPS \\
\hline 39 & Tangible & NA & Low & DLPS \\
\hline 40 & Intangible & Hyperechoic & NA & DLPS \\
\hline 41 & Tangible & NA & Mixed & DLPS \\
\hline 42 & Tangible & Hypoechoic & Low & DLPS \\
\hline 43 & Intangible & NA & Low & DLPS \\
\hline 44 & Tangible & NA & High & DLPS \\
\hline 45 & Intangible & Hypoechoic & Mixed & DLPS \\
\hline 46 & Tangible & NA & Mixed & DLPS \\
\hline 47 & Tangible & NA & Low & DLPS \\
\hline 48 & Tangible & NA & NA & DLPS \\
\hline 49 & Intangible & Hypoechoic & Mixed & DLPS \\
\hline 50 & Tangible & NA & Low & MLPS \\
\hline 51 & Intangible & Hypoechoic & High & MLPS \\
\hline 52 & Tangible & Hyperechoic & Low & MLPS \\
\hline 53 & Tangible & Hypoechoic & Mixed & MLPS \\
\hline 54 & Tangible & Mixed-echoic & Mixed & MLPS \\
\hline 55 & Intangible & Hypoechoic & NA & MLPS \\
\hline 56 & Tangible & NA & Mixed & MLPS \\
\hline 57 & Tangible & Hyperechoic & Mixed & MLPS \\
\hline
\end{tabular}

NA: corresponding examination was not performed.

All 57 patients received surgery, of which 52 (91\%) surgeries were open and $5(9 \%)$ were laparoscopic; all laparoscopic surgeries were performed because of a tumor size less than $10 \mathrm{~cm}$. Besides tumor tissue resection, the invaded organs were also removed during surgery while all nearby uninvolved organs were retained. The most commonly involved organs were the pancreas and kidney. Five (9\%) patients had their pancreas resected; of these, four were pancreas resection, including head, body, and tail, and one had only the head resected. Two (4\%) patients had one-side kidney removals. In 10 patients, the LPS tumor had grown to surround the kidney without direct involvement of the kidney; these tumors were completely resected since a macroscopic fissure existed between the tumor and the kidney. All postsurgical histopathological reports were obtained for analysis; the diagnosis of LPS was confirmed in every patient. Bleeding during surgery ranged from 30 to $8400 \mathrm{~mL}$, with a mean of $910 \mathrm{~mL}$. The $8400 \mathrm{~mL}$ bleeding occurred during separation of the tumor mass from the psoas major, and, unfortunately, we lost contact with this patient two years after surgery. The surgery duration ranged from 2 to 8 hours with a mean of 4.18 hours. Neither surgical bleeding nor duration was related to the size of LPS mass $\left(R^{2}\right.$ for bleeding and size: $0.02, R^{2}$ for duration and size: 0.03 ). Fourteen $(25 \%)$ patients were admitted to the intensive care unit (ICU) in PUMCH for better postsurgical care.
The mean duration of hospital stay was 18.6 (8.9) days (7.6 days prior to surgery and 11.0 days after surgery). For most patients, diagnosis and differential diagnoses such as paraganglioma were the main reasons for the long presurgical hospital stay. In addition, it took some time to fully evaluate the adhesions between the tumor and its neighboring organs, and to further decide on the surgical modality. No significant association was observed between the length of the hospital stay and the size of the LPS (largest diameter greater than $15 \mathrm{~cm}$ by ultrasound or CT) (large vs. small: 17.97 vs. $19.61, P=0.492)$. Postsurgical pathological results confirmed that all patients had LPS, as well as confirming the subtype. During the average 4.5-year followup, $14(25 \%)$ recurrences and $9(18 \%)$ deaths were reported among the 57 patients. Two (4\%) patients received radiotherapy, and 2 (4\%) received chemotherapy following surgery. A complaint of hypoleukemia, lymphopenia, and herpes zoster was received from an 81-year-old female who underwent radiotherapy following surgery. No severe postsurgical complications were reported from other patients, such as hemorrhage or postsurgical infection.

Four patients received postsurgical radiotherapy or chemotherapy, the latter being standard MAID therapy comprising mesna, adriamycin, ifosfamide, and dacarbazine. Recurrence was not observed in any of these four patients during follow-up. At the last follow-up in February 2020, 
there had been $9(16 \%)$ deaths and $14(25 \%)$ recurrences, and we had lost contact with 13 (22\%) patients. According to the Kaplan-Meier survival, DLPS and PLPS with larger sizes and developed symptoms were prone to having lower diseasefree survival, with DLPS having the highest recurrence rate (35\%) and PLPS the highest death rate (25\%). There were no statistically significant differences in prognosis for the invasion of different organs (Figure 3, Table 4).

\section{Discussion}

As a subtype of sarcoma, liposarcoma accounts for approximately $15 \%$ of all sarcomas, making it the most common soft tissue sarcoma [5]. LPS mostly occurs in the extremities, followed by the retroperitoneum. There have also been reports of LPS in rare locations, such as the mediastinum, larynx, or para-testicular tissue [20-22]. The high occurrence in the retroperitoneum may be attributed to metastasis of LPS from other parts of the body, especially those where fat is abundant $[1,2,23]$. Primary retroperitoneal LPS usually originates in the perirenal fat; we observed 19 (33\%) perirenal LPS tumors or instances of LPS directly invading the kidney in our cohort. LPS peaks in the range of 50-60 years, and in our 57-person cohort, the mean age at diagnosis was found to be 57.0 years with 20 (35\%) patients being in $50-60$ age range. We consider this phenomenon to be the result of a higher tolerance in older patients and increased severity in younger patients since PUMCH gathers severe cases of many diseases in China. There was no significant sex difference in LPS occurrence, and the ratio of our cohort was $26(46 \%)$ males to 31 (54\%) females.

The different LPS subtypes have specific genetic mutations. For example, the $\mathrm{t}(12 ; 16)$ (q13;p11) reciprocal translocation results in MLPS $[8,11]$, while gene amplifications in the 12q12-21 and 10p11-14 regions are associated with WDLPS and DLPS, and an additional amplification in either $6 \mathrm{q} 23$ and $1 \mathrm{p} 32$ is also necessary in DLPS $[8,10]$. There are no reports on possible relationships between the occurrence of LPS and exterior factors such as trauma or drug usage.

LPS is usually found accidentally or on regular physical check-ups. The reported clinical symptoms are principally abdominal pain and distension, both in the present cohort and in previously published studies [9, 24-26]. Since abdominal pain and distension are nonspecific and often tolerated by patients, it is difficult to diagnose retroperitoneal LPS or differentiate LPS subtypes via clinical symptoms. We did, however, discover that symptomatic LPS was associated with lower disease-free survival.

In terms of diagnosis using presurgical imaging, $\mathrm{CT}$ and MRI are regarded as the most appropriate modalities. Different subtypes can be distinguished using CT and MRI. WDLPS typically contains more than $75 \%$ adipose tissue with septations thicker than $2 \mathrm{~mm}$ and small internal nodular areas. Using CT, such nodular areas can be found with soft tissue attenuation. Septations and nodular areas in WDLPS show hyperintense character on T2-W1 MRI, distinguishing this subtype from the other LPS types [14]. Although similar to WDLPS, DLPS can still be identified by larger non-lipomatous components containing nodular areas [27]. MLPS often shows a multilobulated, hypoechoic structure on ultrasound [28]. Moreover, MLS usually exhibits low signal intensity in T1W and intermediate signal intensity in T2W, distinguishing it from other types of tumors [12, 25]. PLPS, due to its specific components, shows little fat attenuation on CT [14]. However, hemorrhage and necrosis occur frequently in PLPS, causing heterogeneity on imaging, making diagnosis difficult [29]. Although theoretically distinguishable, no specific subtypes were diagnosed before surgery in this cohort. Because the surgery modality was the same among all subtypes, the presurgical diagnosis of "huge retroperitoneal mass" was enough for surgery. So far, the postsurgery pathological results remain the gold standard for subtype differentiation, [14] allowing predictions of prognosis and the choice of chemotherapy or radiotherapy.

The size of the LPS tumors ranged from $1.2 \times 1.2 \mathrm{~cm}$ to $36.6 \times 26.5 \mathrm{~cm}$ in the present cohort, with a median diameter of $14.6 \mathrm{~cm}$. Using the longest diameter of $15 \mathrm{~cm}$ and $25 \mathrm{~cm}$ as thresholds, the patients were classified into three subgroups in the Kaplan-Meier survival analysis, which demonstrated that the presurgical tumor size had a statistically significant influence on prognosis (Figure 3, Table 4).

Although the extent of resection required is still debatable, surgery is still the key treatment for LPS [30]. Traditionally, a macroscopically negative margin is sufficient for treatment, regardless of pathological subtypes. In our 57patient cohort, all patients received R0 resection with a macroscopically negative resection margin except for two R2 resections, which were both because of older age and poor physical condition. Both patients receiving R2 resections died within one year after surgery. The first $\mathrm{R} 2$ resection, a 75 -year-old female, had a $30 * 15 \mathrm{~cm}$ LPS across her diaphragm and symphysis pubis. The lower half of the LPS was resected successfully, but the upper half was closely adherent to the liver while also surrounding the kidney tightly. Considering that the patient was in poor physical condition, after obtaining the consent of the patient's daughters, the upper half of the LPS was not resected. No adjuvant therapy was applied in this patient. The patient died of LPS 17 months after the surgery. The second R2 resection, an 80 year-old female, had a $40 \mathrm{~cm}$ LPS pushing the duodenum and inferior vena cava to the left side of the abdomen. This patient's LPS had rich blood supply, most of which came from the liver and right kidney. Although the major part of the LPS was resected, the remaining LPS near the liver and right kidney could not be excised. This situation was explained to her relatives, who agreed to an $\mathrm{R} 2$ resection as alleviation. This patient accepted no adjuvant therapy and passed away 13 months after surgery.

The histological subtype is an important factor for prognosis prediction, including local recurrence, distant recurrence, and death. Previous cohort studies have demonstrated that DLPS has the highest risk for both local and distant recurrence, while WDLPS has the lowest risk [19, 31]. In this cohort, we also found that DLPS and PLPS were associated with higher recurrence and malignancy rate (Figure 3 and Table 4) while the recurrence and death rates 


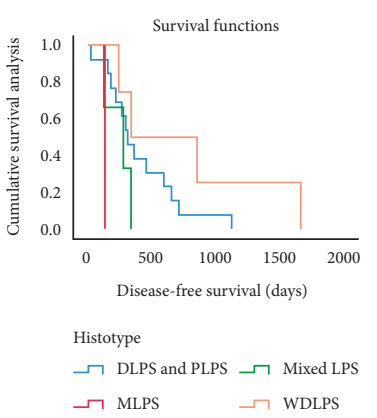

(a)

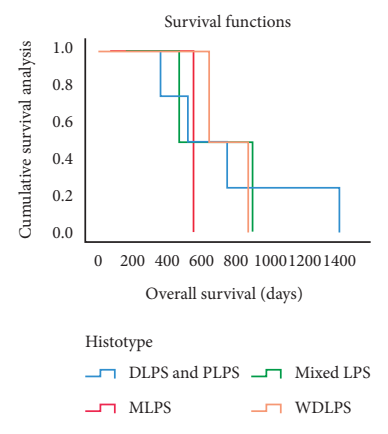

(e)

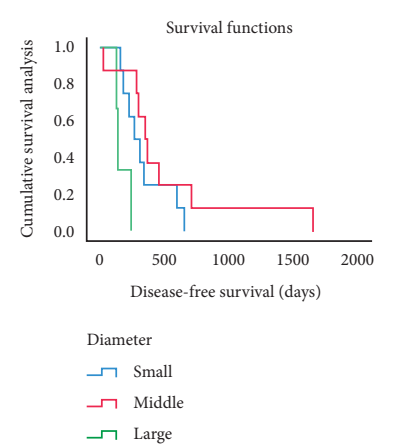

(b)

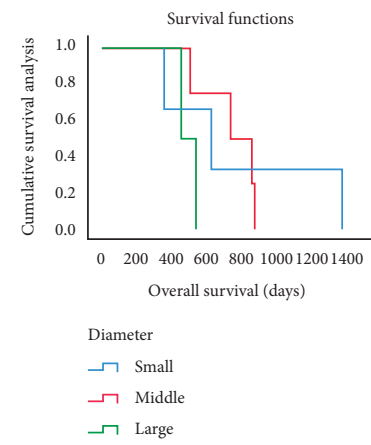

(f)
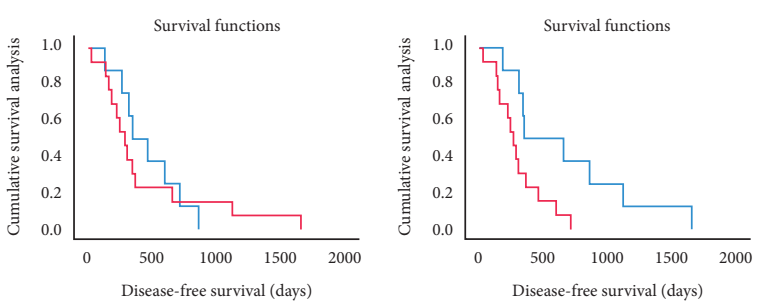

Organ invasion

$\neg$ No

$\neg$ Yes

(c)

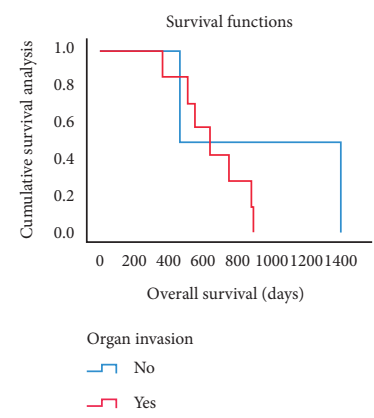

(g)

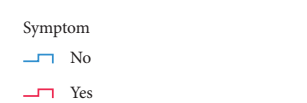

(d)

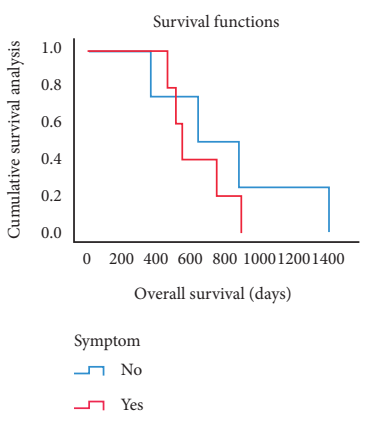

(h)

FIgURE 3: The Kaplan-Meier estimates of DFS and OS of patients with retroperitoneal LPS under different conditions. LPS: liposarcoma; DFS: disease-free survival; OS: overall survival; DLPS: dedifferentiated liposarcoma; PLPS: pleomorphic liposarcoma; MLPS: myxoid liposarcoma; WDLPS: well-differentiated liposarcoma.

TABLE 4: $P$ values of DFS and OS of patients with retroperitoneal LPS under different conditions.

\begin{tabular}{lcccc}
\hline$P$ value & Pathological & Diameter & Organ invasion & Symptom \\
\hline DFS (days) & $P=0.032$ & $P=0.009$ & $P=0.737$ & $P=0.022$ \\
OS (days) & $P=0.930$ & $P=0.298$ & $P=0.375$ & $P=0.466$ \\
\hline
\end{tabular}

LPS: liposarcoma; DFS: disease-free survival; OS: overall survival.

were lowest in the WDLPS subtype, which is consistent with previous reports. All the recurrences reported in our cohort were local. However, we did not identify a relationship between tumor invasion of surrounding organs and postoperative clinical outcomes; this aspect requires further research. The poor prognosis of DLPS encouraged surgeons to explore extended surgery including the en bloc resection of adjacent organs, even though uninvolved $[15,30]$. Studies have shown that extended resection lowers the risk of local recurrence but its effect on overall survival remains unclear $[16,32]$. No extended resections were performed in our cohort, due to concern about poor life quality after adjacent uninvolved organ resection.

There are other approaches to improve LPS prognosis besides the extended surgery modality, such as radiotherapy and chemotherapy. Although retroperitoneal LPS is relatively radiosensitive, so are its nearby organs. An overdose of radiotherapy causes damage to surrounding radiosensitive organs, such as the liver and kidney, so the timing and type of radiotherapy used for retroperitoneal LPS matter [2]. Among all subtypes, MLPS is the most chemo-sensitive, making chemotherapy possible $[12,17,18]$. In our cohort, four patients received postsurgery adjuvant therapy, among which three were diagnosed with MLPS by postsurgery pathological testing. None of the four patients experienced recurrence or death, demonstrating the efficiency of radiotherapy and chemotherapy, but no conclusion can be drawn due to the small sample size. Also, as this is a retrospective study, no standardized postsurgical chemotherapy or radiotherapy was given to these patients. Future guidelines may recommend regular chemotherapy or radiotherapy to postsurgical LPS patients.

There are several limitations to this study. Firstly, as a retrospective study, missing data, recall bias, and errors in the initial medical records may exist. Secondly, the sample size of 57 is relatively small, and the numbers for each subtype are in some cases less than 10. Thirdly, as PUMCH is among the most comprehensive third-grade class- $\mathrm{A}$ hospitals in China, the high number of severe and difficult surgical cases seen may give rise to bias.

We collected and analyzed the detailed demographic and clinical data of all 57 patients. We found that presurgery 
imaging helped to diagnose LPS, and specific subtypes could also be distinguished via CT or MRI. The prognosis for the different subtypes differed. Recurrence and death occurred more frequently in symptomatic patients with larger DLPS and PLPS tumors; in contrast, WDLPS had relatively low recurrence and death rates. A macroscopically negative margin was the surgical goal for most of the patients in this cohort, and an aggressive surgery modality to resect adjacent uninvolved organs has also been proposed by other researchers. Radiotherapy and chemotherapy may further improve prognosis. Generally, this cohort has helped deepen our understanding of LPS and describes the characteristics of a Chinese retroperitoneal LPS cohort.

\section{Summary}

We collected and analyzed the available data from 57 retroperitoneal myxoid liposarcoma patients over an average follow-up time of 4.5 years. Using analysis of current data and comparison with previous studies, we have identified key factors concerning presurgery diagnosis as well as factors influencing prognosis and treatment. The analysis of clinical symptoms, imaging, including CT, ultrasound, and MRI, provides critical evidence when diagnosing LPS and its subtypes. The most important factors deciding prognosis include the LPS subtype, presurgical LPS size, and whether the patient is symptomatic. Among all subtypes, WDLPS had the best prognosis while DLPS and PLPS had the worst. $\mathrm{R} 0$ resection is the key treatment for all subtypes, and an aggressive surgery modality to resect uninvolved adjacent organs in DLPS and chemotherapy and radiotherapy for MLPS are also alternative choices.

\section{Abbreviations}

MLS: $\quad$ Myxoid liposarcoma

PE: $\quad$ Physical examination

PUMCH: Peking Union Medical College Hospital

CT: $\quad$ Computed tomography

MRI: $\quad$ Magnetic resonance imaging

FUS: $\quad$ Fused in sarcoma

DNA: Deoxyribonucleic acid

SD: $\quad$ Standard deviation

ICU: Intensive care unit.

\section{Data Availability}

The data used to support the findings of this study are available from the corresponding author upon request.

\section{Disclosure}

Jianchun Xiao and Jianghao Liu should be considered as cofirst authors. Minting Chen is the second author.

\section{Conflicts of Interest}

The authors declare that they have no conflicts of interest.

\section{Authors' Contributions}

Jianchun Xiao and Jianghao Liu contributed equally to this work.

\section{Acknowledgments}

National Key Research and Development Program (Grant no. 2020YFF0305104).

\section{References}

[1] W. W. Huh, C. Yuen, M. Munsell et al., "Liposarcoma in children and young adults: a multi-institutional experience," Pediatric Blood \& Cancer, vol. 57, no. 7, pp. 1142-1146, 2011.

[2] A. Vijay and L. Ram, "Retroperitoneal liposarcoma," American Journal of Clinical Oncology, vol. 38, no. 2, pp. 213-219, 2015.

[3] A. Sonoda, H. Sawayama, N. Miyanari, T. Mizumoto, T. Kubota, and H. Baba, "Giant myxoid liposarcoma of the stomach: report of a case," International Journal of Surgery Case Reports, vol. 60, pp. 234-238, 2019.

[4] O. Uslukaya, F. Taskesen, I. Aliosmanoglu, Z. Arikanoglu, M. Gul, and A. Dusak, "Giant myxoid liposarcoma of descending mesocolon origin," Gastroenterology Review, vol. 9, no. 6, pp. 361-364, 2014.

[5] E. Grasso, F. Marino, M. Bottalico, and M. Simone, "A case of myxoid liposarcoma of the retroperitoneum: a challenging tumour for diagnosis and treatment," Case Reports in Surgery, vol. 2014, Article ID 572805, 2014.

[6] R. S. A. De Vreeze, D. De Jong, and I. H. G. Tielen, "Primary retroperitoneal myxoid liposarcomas," Modern Pathology, vol. 22, no. 2, pp. 223-231, 2009.

[7] A. T. J. Lee, K. Thway, P. H. Huang, and R. L. Jones, "Clinical and molecular spectrum of liposarcoma," Journal of Clinical Oncology, vol. 36, no. 2, pp. 151-159, 2018.

[8] L. Yang, S. Chen, P. Luo, W. Yan, and C. Wang, "Liposarcoma: advances in cellular and molecular genetics alterations and corresponding clinical treatment," Journal of Cancer, vol. 11, no. 1, pp. 100-107, 2020.

[9] K. Thway, "Well-differentiated liposarcoma and dedifferentiated liposarcoma: an updated review," Seminars in Diagnostic $\mathrm{Pa}$ thology, vol. 36, no. 2, pp. 112-121, 2019.

[10] F. Pedeutour, G. Maire, A. Pierron et al., "A newly characterized human well-differentiated liposarcoma cell line contains amplifications of the 12q12-21 and 10p11-14 regions," Virchows Archiv, vol. 461, no. 1, pp. 67-78, 2012.

[11] J. S. E. Yu, S. Colborne, C. S. Hughes, G. B. Morin, and T. O. Nielsen, "The FUS-DDIT3 interactome in myxoid liposarcoma," Neoplasia, vol. 21, no. 8, pp. 740-751, 2019.

[12] S. Abaricia and A. C. Hirbe, "Diagnosis and treatment of myxoid liposarcomas: histology matters," Current Treatment Options in Oncology, vol. 19, no. 12, p. 64, 2018.

[13] C. J. Chouairy, F. W. Abdul-Karim, and G. T. MacLennan, "Retroperitoneal liposarcoma," Journal of Urology, vol. 177, no. 3, p. 1145, 2007.

[14] O. Teniola, K. Y. Wang, W.-L. Wang, W. W. Tseng, and B. Amini, "Imaging of liposarcomas for clinicians: characteristic features and differential considerations," Journal of Surgical Oncology, vol. 117, no. 6, pp. 1195-1203, 2018.

[15] M. Fairweather, R. J. Gonzalez, D. Strauss, and C. P. Raut, "Current principles of surgery for retroperitoneal sarcomas," Journal of Surgical Oncology, vol. 117, no. 1, pp. 33-41, 2018.

[16] A. Gronchi, S. Lo Vullo, M. Fiore et al., "Aggressive surgical policies in a retrospectively reviewed single-institution case 
series of retroperitoneal soft tissue sarcoma patients," Journal of Clinical Oncology, vol. 27, no. 1, pp. 24-30, 2009.

[17] M. Saponara, S. Stacchiotti, and A. Gronchi, "Pharmacological therapies for liposarcoma," Expert Review of Clinical Pharmacology, vol. 10, no. 4, pp. 361-377, 2017.

[18] L. P. Suarez-Kelly, G. G. Baldi, and A. Gronchi, "Pharmacotherapy for liposarcoma: current state of the art and emerging systemic treatments," Expert Opinion on Pharmacotherapy, vol. 20, no. 12, pp. 1503-1515, 2019.

[19] S. Singer, C. R. Antonescu, E. Riedel, and M. F. Brennan, "Histologic subtype and margin of resection predict pattern of recurrence and survival for retroperitoneal liposarcoma," Annals of Surgery, vol. 238, no. 3, pp. 358-371, 2003.

[20] Y. S. Yang, C. Y. Bai, Z. C. Li, W. J. Li, and Y. Li, "Giant primary liposarcoma of the anterior mediastinum: a case report," Medicine (Baltimore), vol. 97, no. 42, 2018.

[21] Y. Han, L.-H. Yang, and T.-T. Liu, "Liposarcoma of the larynx report of a case and review of literature," International Journal of Clinical and Experimental Pathology, vol. 8, no. 1, pp. 1068-1072, 2015.

[22] K. Mouden, S. Wakrim, and A. Semmar, "Paratesticular liposarcoma: a case report," Pan African Medical Journal, vol. 33, p. 282, 2019.

[23] K. Sheah, H. A. Ouellette, M. Torriani, G. P. Nielsen, S. Kattapuram, and M. A. Bredella, "Metastatic myxoid liposarcomas: imaging and histopathologic findings," Skeletal Radiology, vol. 37, no. 3, pp. 251-258, 2008.

[24] K. Thway, R. L. Jones, J. Noujaim, S. Zaidi, A. B. Miah, and C. Fisher, "Dedifferentiated liposarcoma updates on morphology, genetics, and therapeutic strategies," Advances in Anatomic Pathology, vol. 23, no. 1, pp. 30-40, 2016.

[25] H. R. Durr, J. Rauh, A. Baur-Melnyk et al., "Myxoid liposarcoma: local relapse and metastatic pattern in 43 patients," BMC Cancer, vol. 18, no. 1, p. 304, 2018.

[26] L. Wang, R. Luo, Z. Xiong, J. Xu, and D. Fang, "Pleomorphic liposarcoma: an analysis of 6 case reports and literature review," Medicine (Baltimore), vol. 97, no. 8, 2018.

[27] M. D. Murphey, L. K. Arcara, and J. Fanburg-Smith, "From the archives of the AFIP imaging of musculoskeletal liposarcoma with radiologic-pathologic correlation," Radiographics, vol. 25, no. 5, pp. 1371-1395, 2005.

[28] J. P. Jagannathan, S. H. Tirumani, and N. H. Ramaiya, "Imaging in soft tissue sarcomas," Surgical Oncology Clinics of North America, vol. 25, no. 4, pp. 645-675, 2016.

[29] W. D. Craig, J. C. Fanburg-Smith, L. R. Henry, R. Guerrero, and J. H. Barton, "Fat-containing lesions of the retroperitoneum radiologic-pathologic correlation," Radiographics, vol. 29, no. 1, pp. 261-290, 2009.

[30] S. A. Mansfield, R. E. Pollock, and V. P. Grignol, "Surgery for abdominal well-differentiated liposarcoma," Current Treatment Options in Oncology, vol. 19, no. 1, p. 1, 2018.

[31] E. K. Bartlett, C. E. Curtin, K. Seier et al., "Histologic subtype defines the risk and kinetics of recurrence and death for primary extremity/truncal liposarcoma," Annals of Surgery, vol. 10, 2019.

[32] S. Bonvalot, M. Rivoire, M. Castaing et al., "Primary retroperitoneal sarcomas: a multivariate analysis of surgical factors associated with local control," Journal of Clinical Oncology, vol. 27, no. 1, pp. 31-37, 2009. 